\title{
Compliance with National Snakebite Treatment Guidelines in Rural Sri Lankan Hospitals: A Cluster Randomized Controlled Trial of a Brief Educational Intervention
}

Seyed Shahmy ( $\nabla$ shahmy_phr@yahoo.com )

National Science \& Technology Commission of Sri Lanka

Senanayake AM Kularatne

University of Peradeniya

Indika B Gawarammana

University of Peradeniya

Shantha S Rathnayake

South Asian Clinical Toxicology Research Collaboration

Andrew H Dawson

University of Sydney

\section{Research Article}

Keywords: Cluster RCT, Educational Intervention, Health care workers, Primary Hospitals, Treatment guidelines, Snakebites, Sri Lanka

Posted Date: March 1st, 2022

DOI: https://doi.org/10.21203/rs.3.rs-968138/v1

License: (c) (i) This work is licensed under a Creative Commons Attribution 4.0 International License.

Read Full License 


\section{Abstract}

\section{Background}

Snakebite is a global health problem predominately occurring in rural areas. In Sri Lanka, the majority of snakebites first present to smaller rural primary hospitals. Improving care delivered at rural hospitals has the potential to reduce morbidity and mortality from snakebites.

\section{Objective}

In this study, we evaluated whether an educational intervention would increase compliance with national snakebite treatment guidelines in primary hospitals.

\section{Methods}

The hospitals were randomized into educational intervention $(n=24)$ and control group $(n=20)$. The intervention hospitals received a brief educational intervention based on Sri Lankan Medical Association (SLMA) guidelines on management of snakebites. Control hospitals had free access to the guidelines but no additional promotion. Four outcomes were assessed; Pre and Post-test knowledge at the completion of a one-day workshop of educational intervention (Intervention group only), Improvement of quality of the patients' medical records, appropriateness of transfers to higher hospitals, and quality of overall management graded by a blinded expert. The data were collected for a period of 12 months.

\section{Results}

All case notes of snakebite hospital admissions were reviewed. There were 1021 cases in the intervention and 1165 cases in the control hospitals. Four hospitals in the intervention and 03 hospitals in the control groups did not have snakebite admissions and were excluded from the cluster analysis. The absolute quality of care was high in both groups. Post-test knowledge was improved $(p<0.0001)$ following the intervention group's educational workshop. There was no statistical difference between the two groups in the documentation of clinical data in hospital notes (scores, $p=0.58$ ), appropriateness of transfers $(p=0.68)$ both of which were at significant variance to the guidelines

\section{Conclusion}

Representative group education from primary hospitals had improved the immediate knowledge gaining. However, that failed to influence the record-keeping and treatment decisions, as all primary hospitals showed adequate compliance with the existing SLMA guidelines.

\section{Trial registration}

The study was registered with Sri Lanka Medical Associations' clinical trial registry https://slctr.lk/trials . Reg. No SLCTR/2013/023. Registered: 30/07/2013. 


\section{Background}

Snakebite is considered a neglected tropical disease[1]. In Sri Lanka, there are approximately 40,000 hospital admission following snakebites reported annually[2],[3].In a nationwide community based study which included snakebite victims who did not present to hospital there was more than 80,000 bites annually[4]. Most of the envenoming results from the land snakes such as Russell's viper (Daboia russelii), Hump-nosed pit viper (Hypnale species), Common krait (Bungarus caeruleus), Saw-scaled viper (Echis carinatus) and Cobra (Naja naja) [5],[6],[7].In the year 2018, the overall in hospital mortality from snakebite was 1.63 per 1000 admissions [2] and there were 464 deaths in Sri Lanka [4]. The majority of snakebites present to primary hospitals of the island manned by non-specialist medical doctors. A sizable proportion of patients are then transferred to secondary or tertiary care hospitals where specialists are available for further care [3]. Thus, primary care hospital plays a pivotal role in clinical management of patients that decides the burden of morbidity and mortality in snakebite.

The Sri Lanka Medical Association (SLMA), the apex body in medicine in Sri Lanka has a lead role in advocacy for management of snakebite including development of management guidelines[8], a hotline service and liaison with the health authorities. Understanding how well these guidelines are used in primary care clinical practice is important for both patient care and subsequent guideline development.

The objective of this study was to test whether an additional systematic educational program (educational intervention) based on SLMA national guideline improves knowledge and clinical practice of first contact doctors and nurses of primary care hospitals compared with the access to freely available online guidelines alone.

\section{Methods}

\section{Study design and Enrollment}

We conducted a cluster randomized control trial in the predominately rural Kurunegala district of the North Western Province of Sri Lanka. The district has 44 primary hospitals with in-patients' facilities that were eligible and randomized to the study. The primary hospitals included 4 larger base hospitals with subspecialty care and 40 smaller divisional hospitals with lower resources and no specialist care. Control hospitals had free access to guidelines whilst intervention hospitals received a brief educational intervention. Following the completion of the educational intervention, snakebite admissions of all enrolled hospitals were collected for one year between 25th May 2013 and 25th May2014.

\section{Sample Size}

The study was a convenience sample which included all patients with the history of snakebites presented to the 44 study hospitals for the period of one year from 25th May 2013 to 25th May 2014 for the analysis. The convenience sampling is the most applicable and widely used method in clinical research and the convenient and purposive samples are important and necessary as long as the readers are aware 
about of the populations to which the findings are relevant[9],[10]. We hypothesized that including all the snakebites admitted to the entire study hospitals for one year avoid biases.

\section{Randomization}

The cluster randomization was done by an independent statistician with the individual unit of randomization was by hospital. The cluster randomization was stratified by including the following variables: government category of hospitals (reflecting hospital size and facilities), number of snakebite admission and geographical areas of the hospital in relation to Kurunegala district. Finally, 24 hospitals were randomized to intervention group and 20 hospitals to the control group.

\section{Interventions}

A full day interactive teaching session (to resemble the train- the- trainer mode) including group discussions, lectures, role play, and demonstration were conducted at a central location in the district. The workshop was designed and delivered by experts covering the topics such as identification of the offending snakes, epidemiology and manifestations of envenoming, complications, appropriate transfer referrals, appropriate use of antivenom (AV) and management of $A V$ reactions and resuscitation as per SLMA guidelines. At the beginning and end of the session, participant's knowledge was evaluated through pre- and post-respectively. The expert panel consisted of locally known senior consultant physicians, the principal researchers and a herpetologist.

The participants to the intervention workshop were encouraged to disseminate the gained knowledge and skills learned through the sessions to the rest of staff in their treating facilities at the earliest possible time. To make sure this activity, the research team visited each intervention hospital, within a period of one week from the day of the workshop, to facilitate the intended interactive discussions between the session participants and the rest of the medical staff at their respective treating facilities. In line with this, the entire management team of each hospital in the intervention group was invited to either a ward setup or a doctors rest room for a guided discussions. The study team provided the multimedia and other facilities to convene these interactive discussions. If any staff were absence during these occasions due to their commitments, the arrangements were done to brief them on the subject at another day as per convenient to them. The procedure was reinforced throughout the period of the study.

Each intervention hospital received the SLMA treatment guideline[8], user friendly patient medical record known as a Bed Head Ticket (BHT) folio annexures (Additional File 1_ BHT_folio), wall poster depicting pictures of deadly venomous snakes and essential management steps depicted in an algorithm. This was supported by promotional items such as pens, T-Shirts with printed messages promoting the use of the national guidelines (Table 1 ). The study team routinely monitored to make sure their availability and use in the hospitals in the intervention group.

The control group did not participate in the workshop and received neither the printed version of freely available online SLMA treatment guidelines nor any other components of the intervention. Also study team did not have any interaction with the management team on education. 


\section{Preparation and validation of components of the intervention (at Pre-intervention period) \\ Preformatted BHT folio annexures}

The initial draft of the pre-formatted BHT folios were prepared by a team of resource personals consisted of locally known senior consultant physicians. Then, a group of medical practitioners representing from primary health care units were invited in conjunction with provincial health treating authority to a central location to review the format of the folios. Subsequently, the revised version was circulated to a number of primary hospitals to test it under practical scenario and accordingly, the changes were adapted to finalize the version which would be taken for the intervention.

\section{Educational Charts}

The initial version of the wall posters depicting pictures of deadly venomous snakes and essential management steps depicted in an algorithm were prepared by a content specialist in par with the guidelines of SLMA snakebite management and circulated to a group of locally known senior clinicians and to a herpetologist for content verification.

\section{Data collection and data management}

The data extracted from the patients' hospital records were transcribed into pre structured data extraction forms (Additional File 2_Data Extraction form), and entered into a database in Microsoft access by trained research assistants. The main variables extracted were demographic information, identification of snakes, clinical signs, reasons for the transfers and treatment. The patients' records were scanned and linked to the patient's database record.

The database entry was independently cross checked with the scanned patient record for accuracy and completeness by two other researchers. Data linkage was undertaken to identify the outcome of any patient transferred from a primary hospital to the Teaching Hospital, Kurunegala (THK). After data linkage for transfers, all subsequent analysis was anonymized.

The details of the hospital stocking of antivenom, the contents of the resuscitation trolley were checked routinely for the availability of bag valve mask (Ambu bags) and test tubes to conduct the 20 minuteWhole Blood Clotting Time (WBCT20) test, which has been used for decades on identifying the clinically significance coagulopathy in snakebites [11].

\section{Ethics Statement}

The study was approved by the Ethical Review Committee, Faculty of Medicine, University of Peradeniya, Sri Lanka. As the study was undertaken in collaboration with the treating health authority, North Western Province, individual patient consent was not required by the Institutional Review Board (IRB). The protocol 
for this trial and supporting CONSORT checklist are available as related manuscript files; see Additional File 4_Study _Protocol and Additional File 5_CONSORT_Checklist_ClusterRCT

\section{Outcomes and Assessments}

The pre and post-tests of pre structured multiple-choice questionnaires (MCQ) were evaluated to check the gained knowledge at the beginning and at the end of the day of teaching session, respectively.

The primary outcomes were the: post interventional improvement of documentation in the hospital records (BHT), number of appropriate transfers, overall patient management graded by an expert clinician who was blinded to the study randomization.

Overall patient management comprised of accuracy of identification of offending snakes; evidence of envenoming; indication for antivenom (AV) therapy; regimen and dose of AV therapy and management of $\mathrm{AV}$ adverse reactions. The secondary outcomes of mortality at different levels and the audit of morbidity of transferred cases were evaluated at referral hospitals.

\section{Expert review}

Using 24 predefined criteria (Additional File 3_24 Predetermined_Points) based on the SLMA guideline [8]an expert clinician independently reviewed and scored the scanned copies of the primary hospitals records to identify the appropriateness of transfers to the tertiary care centers and of overall patient management. The "Satisfactory" level for overall patient management had to fulfill all the following criteria where applicable; the correct identification of the type of bite, correct identification of envenomation features with respect to the bites, and the clinical management of them, correct indication for AV, and the identification and the management of complications (of snakebite or AV). The clinician was blind to the randomization status of the hospital. The tertiary hospital records were also reviewed for those patients who were transferred from a primary hospital. The accepted indications of transfers were anticipated ventilatory problems, need of ICU care, severe coagulopathy, impending acute kidney injury, need of surgical care for local necrosis and refractory shock, lack of resuscitation facilities, AV and emergency medications.

\section{Statistical Analysis}

The data were analysed using $\mathrm{R}$ software statistical package version 3.2.5, through the packages aod[12] and clusrank[13].

Median and IQR was calculated for pre and post-test and Wilcoxon signed rank test performed to compare the scores between them (Paired samples). The Clustered Wilcoxon rank sum test was performed to compare the scores between the quality of the patients medical record between the two groups(independent samples)[14],which was assessed for 24 pre determinant points. Test of proportion of homogeneity for appropriate transfers, if the patient was managed correctly at primary hospitals was performed through Donner ICC adjusted Chi-squared test [15],[16].The $p$ value with $<0.05$ was regarded as statistically significant. 


\section{Results}

There were 2 Base Hospitals and 22 Divisional Hospitals in the intervention group. Two to 3 health care members (composed of first contact doctors and nurses) participated at the workshop as representatives of each hospital. The proportion of staff attending training was higher for Divisional hospital [Median number of doctors working in the divisional hospitals was 3 and nursing officers was 6] than Base hospitals which have much greater staff numbers.

Fifty participants from interventional hospitals participated in the training workshop. Pre-test results for initial evaluation were available for all the participants. For the subsequent analysis, only 34 post test results were available as 16 participants have left the workshop just before the post-test to attend their official duties or personal commitments. However, they were present during the whole workshop which ran into both morning and afternoon of the day. It was understood that they were working in limited resource setup with meager man power in faraway places. After the workshop, the Knowledge had improved significantly from a pre-test median score of 16/40(IQR: 10.25 -20) to post-test median score of 23.5/40 (IQR: 18.25-28) $(p<0.00001)$

After one year of follow up, the primary analysis included 1021 snakebite cases from 20 intervention hospitals and 1165 cases from 17 control hospitals. There were 107 patients (10.5\%) in the intervention group and $142(12 \%)$ patients in the control group reported with comorbid-medical conditions, the total number of Co-morbidities were reported either as sole manifestations or in combination, without the differences in patient baseline characteristics of age, gender and type of bites reported (Table 2 and 3 ). There were no deaths reported from both the groups in the primary hospitals or subsequently transfer to the tertiary care units. The details of the hospitals outcomes of the patients are given in Table 4.

Four hospitals in the intervention and 03 hospitals in the control groups did not have cases of snakebite and subsequently excluded from the cluster analysis (Figure 1).

\section{Primary outcomes}

There was no difference between the groups in any of the 3 primary outcomes of improvement of quality of patient records (Figure 2) appropriateness of transfers and overall patient management (Table 5). Overall clinical management was high in both groups. The quality of documentation in the BHT was poor and rates of inappropriate transfer were high in both groups.

There were 208 (20.4\%) patients transferred out of the primary hospitals in the intervention group, while $191(16.4 \%)$ cases in the control group. Whether the transfer decisions were appropriate or not based on records are shown in Figure 3 , with the appropriates transfers as $15 \%(32 / 208)$ vs. $14 \%(26 / 191)$ respectively. Of these, 294 cases were transferred to the district tertiary care unit of Teaching Hospital Kurunegala (THK) and 105 patients to a facility in another health district. Of the transfer cases to THK, 93 $(60.4 \%)$ case records in intervention group and $84(60 \%)$ case records in control group were available for subsequent analysis. A subgroup analysis of the decision of transfer to THK as appropriate or not is 
shown in Figure 4, with 17\% vs. 10 \% respectively. Of these, 30 (17\%) cases received AV at THK (19 cases in intervention Vs. 11 cases in control group). Of them, 14 cases were identified by the expert review as having indications for AV in their respective primary hospitals ( 9 in intervention Vs. 5 in control group).

\section{Discussion}

There were no deaths within the entire cohort. Rates of overall satisfactory treatment including appropriate identification and transfer decisions of high-risk cases was high in both study arms (>90\%). This suggests the combination of prior education, experience and 10 years of SLMA national guidelines have been effective in addressing the core clinical challenges of snakebite in Sri Lanka.

Disappointingly, the educational intervention had no impact on high rates of inappropriate inter-hospital transfers occurring without medical indication and outside of the current guidelines in both groups. The cost of inter-hospital transfer is a significant component of total patient cost in Sri Lanka[17]. The cost of hospital transfer accounted for almost half of the total treatment costs at primary hospital level in pesticide poisoning in Sri Lanka [18]. Given the high quality of overall management in the primary hospitals this paradoxical result requires further investigation to refine a more effective intervention. Within our study, lack of local resources did not explain transfer rates. A previous study has shown that medical decisions in primary hospitals may be influenced both by non-medical personal and community expectations [17] in a context where doctors may feel isolated[19]. Doctors in primary care hospitals are in direct contact with the public and their image and reputation is at stake if a death happen under his or care. Future interventions to address transfer rates should highlight the success of primary hospital management of snakebite demonstrated in this study. Such interventions may need to be directed to the local community as well as medical staff.

There was no difference in the quality of documentation between the groups. There are a number of explanations for this and need to address in the context of the high rates of overall clinical compliance. The first is that some of scored items may have little clinical relevance such as patient education and occupation details in determining individual patient management. While structured admission forms for toxicology have been shown to improve documentation [20], there has to be a perceived benefit for the clinician in using such forms. Such a benefit may not be perceived by an individual doctor working remotely who is not providing clinical information to others outside of the primary hospital.

As over $80 \%$ of snakebites in Sri Lanka are treated in primary hospitals[3] it is important to ensure that management is of high quality and designed to be delivered within these clinical settings. The current results support that this is being achieved. For many years, there has been relatively little change in snakebite treatment in Sri Lanka but it is important to have mechanisms that can effectively propagate clinical change if it occurs. The most likely change in Sri Lanka will be the introduction of new antivenom with different doses and indications for use. Brief educational interventions have been previously successful in promoting guideline change in Sri Lanka[21]. Further research on how to disseminate 
training to small institutions needs to be done but a train the trainer approach appears to be effective [22].

\section{Limitation}

With such a high baseline rate of appropriate care our study was underpowered to detect any improvement in clinical care. Anecdotally, we became aware that some educational materials has filtered to control group hospitals due to possible transfers of the medical staff within the study district might have taken place during the period of the study and also SLMA hotline services on advocacy for snakebite management might be utilized by the treating physicians at control group hospitals.

\section{Conclusion}

Overall treatment quality of snakebite is satisfactory in primary care hospital in NWP. The brief educational intervention did not change the established practice in all primary hospitals where there is no restriction to access of SLMA guidelines. Therefore, we recommend that SLMA continue to update guideline and make it available to all hospitals in Sri Lanka

\section{Abbreviations}

Ambu bags: Bag Valve Mask

AV: Antivenom

BHT: Bed Head Ticket

ICC: Intraclass Correlation Coefficient

LAMA: Left Against Medical Advice

NWP: North Western Province of Sri Lanka

IRB: Institutional Review Board

MCQ: Multiple Choice Questions

SLMA: Sri Lanka Medical Association

THK: Teaching Hospital Kurunegala

WBCT20- 20 Minute Whole Blood Clotting Time

\section{Declarations}




\section{Ethics Approval and Consent to Participate}

The study was approved by the Ethical Review Committee, Faculty of Medicine, University of Peradeniya, Sri Lanka. As the study was undertaken in collaboration with the treating health authority, North Western Province, individual patient consent was not required by the Institutional Review Board (IRB). The protocol for this trial and supporting CONSORT checklist are available as related manuscript files; see Additional File 4_Study _Protocol and Additional File 5_CONSORT_Checklist_ClusterRCT

\section{Consent for Publication}

Not Applicable

\section{Availability of data and material}

All data generated or analysed during this study are included in this published article (and its Additional supplementary Information files).

\section{Competing interests}

The authors declare no competing interests

\section{Funding}

This study was supported by Australian NHMRC grants 630650, 1055176 and 1110343

\section{Authors' contributions}

SAMK and AHD conceived the research idea and designed the study; all authors performed further development; SS, SAMK, SSR and IGB conducted the experiments; SS and SAMK analysed the data and drafted the manuscript; AHD assisted in data analysis and edited the manuscript; all authors contributed to the final version of the manuscript and take responsibility for the manuscript.

\section{Acknowledgments}

We thank the staff of study hospitals in the Kurunegala district and Provincial Director of Health Services -NWP Sri Lanka for their invaluable support.

We would also thankful to Dr.Wasantha Dissanayake- Consultant Physician, THK, Dr. Hemantha Rajapaksha - Consultant Anesthetist THK and Dr. Anslem De Silva-Herpetologist for taking part as 
resource personals to conduct the interactive work shop. Also, we wish to thank the SACTRC project team including Ms. Thilini Siriwardhana (Project Manager), and Ms. Miulika Rupasingha (Coordinating Secretary) for their given support to run the program smoothly and Dr. Inoka Gunathilake for assistance in data entry.

\section{References}

1. Warrell DA. Guidelines for the management of snake-bites. Guidelines for the management of snakebites. 2010.

2. Ministry of Health S. Annual Health Bulletin In: Unit MS, editor.: Ministry of Health; 2018. p. 237.

3. Shahmy S, Kularatne SA, Rathnayake SS, Dawson AH. A prospective cohort study of the effectiveness of the primary hospital management of all snakebites in Kurunegala district of Sri Lanka. PLoS neglected tropical diseases. 2017;11(8):e0005847.

4. Ediriweera DS, Kasturiratne A, Pathmeswaran A, Gunawardena NK, Wijayawickrama BA, Jayamanne SF, et al. Mapping the risk of snakebite in Sri Lanka-a national survey with geospatial analysis. PLoS neglected tropical diseases. 2016;10(7):e0004813.

5. Alirol E, Sharma SK, Bawaskar HS, Kuch U, Chappuis F. Snake bite in South Asia: a review. PLoS Negl Trop Dis. 2010;4(1):e603.

6. Kasturiratne A, Pathmeswaran A, Fonseka M, Lalloo D, Brooker S, de Silva H. Estimates of disease burden due to snakebite in Sri Lankan hospitals. 2003.

7. Kularatne S. Clinical profile of snake envenoming: a study in North Central Province of Sri Lanka. The 23rd bibile memorial oration in 2001. Sri Lanka J Med. 2001;10:4-12.

8. Association ECoSSLM. GUIDLINES FOR THE MANAGEMENT OF SNAKEBITE IN HOSPITAL SLMA Colombo 07: SLMA; 2013 [updated March 2013; cited 2013]. Electronic Guidlines Vesrion 3.0:2013 revised and expanded:[Available from: http://slma.Ik/wp-content/uploads/2014/12/Guidelines-formanagement-of-snake-bite-in-hospital.pdf.

9. Andrade CJIJoPM. The inconvenient truth about convenience and purposive samples. 2020:0253717620977000.

10. Elfil M, Negida AJE. Sampling methods in clinical research; an educational review. 2017;5(1).

11. Ratnayake I, Shihana F, Dissanayake DM, Buckley NA, Maduwage K, Isbister GK. Performance of the 20-minute whole blood clotting test in detecting venom induced consumption coagulopathy from Russell's viper (Daboia russelii) bites. Thrombosis and haemostasis. 2017;117(03):500-7.

12. Lesnoff M, Lancelot, R. aod: Analysis of Overdispersed. 2012.

13. clusrank: Wilcoxon Rank Sum Test for Clustered [Internet]. Data. R package version 0.5-2. 2017. Available from: https://CRAN.R-project.org/package=clusrank.

14. Rosner B, Glynn RJ, Lee MLT. Extension of the Rank Sum Test for Clustered Data: Two-Group Comparisons with Group Membership Defined at the Subunit Level. Biometrics. 2006;62(4):1251-9. 
15. Donner A. Statistical methods in ophthalmology: an adjusted chi-square approach. Biometrics. 1989:605-11.

16. Jeong KM. Tests for homogeneity of proportions in clustered binomial data. Communications for Statistical Applications and Methods. 2016;23(5):433-44.

17. Wickramasinghe K, Steele P, Dawson A, Dharmaratne D, Gunawardena A, Senarathna L, et al. Cost to government health-care services of treating acute self-poisonings in a rural district in Sri Lanka. Bulletin of the World Health Organization. 2009;87(3):180-5.

18. Ahrensberg H, Madsen LB, Pearson M, Weerasinghe M, Eddleston M, Jayamanne S, et al. Estimating the government health-care costs of treating pesticide poisoned and pesticide self-poisoned patients in Sri Lanka. 2019;12(1):1692616.

19. Senarathna L, Hunter C, Dawson AH, Dibley MJ. Social dynamics in rural Sri Lankan hospitals: Revelations from self-poisoning cases. Qualitative health research. 2013;23(11):1481-94.

20. Buckler NA, Whyte IM, Dawson AH, Reith DA. Preformatted admission charts for poisoning admissions facilitate clinical assessment and research. Annals of emergency medicine. 1999;34(4):476-82.

21. Senarathna L, Buckley NA, Dibley MJ, Kelly PJ, Jayamanna SF, Gawarammana IB, et al. Effect of a brief outreach educational intervention on the translation of acute poisoning treatment guidelines to practice in rural Sri Lankan hospitals: a cluster randomized controlled trial. PloS one. 2013;8(8):e71787.

22. Rajapakse BN, Neeman T, Dawson AH. The effectiveness of a 'train the trainer'model of resuscitation education for rural peripheral hospital doctors in Sri Lanka. PLoS One. 2013;8(11):e79491.

\section{Tables}

Table 1 is available in the Supplemental Files section. 
Table 2

Baseline Characteristics of Snakebite patients admitted to study Hospitals in Kurunegala District of Sri Lanka.

\begin{tabular}{|lll|}
\hline & $\begin{array}{l}\text { Intervention } \\
\mathbf{n}(\%)\end{array}$ & $\begin{array}{l}\text { Control } \\
\mathbf{n}(\%)\end{array}$ \\
\hline Gender & $606(59.3)$ & $683(58.6)$ \\
$\cdot$ Male & $415(40.6)$ & $482(41.3)$ \\
\hline Age groups & & \\
$\cdot<12$ & $58(5.7)$ & $81(6.9)$ \\
$\cdot$ 12-19 & $88(8.6)$ & $98(8.4)$ \\
$\cdot$-20-29 & $143(14)$ & $156(13.4)$ \\
$\cdot$ 30-39 & $190(18.6)$ & $210(18)$ \\
$\cdot$ 40-49 & $202(19.8)$ & $225(19.3)$ \\
$\cdot>=50$ & $340(33.3)$ & $395(33.9)$ \\
\hline Type of Bite & $30(2.9)$ & $31(2.7)$ \\
$\cdot$ Russell's Viper & $414(40.5)$ & $409(35)$ \\
$\cdot$ Hump-nosed Viper & $3(0.3)$ & $11(1)$ \\
$\cdot$ Cobra & $5(0.5)$ & $8(0.7)$ \\
$\cdot$ Common Krait & $2(0.2)$ & $1(0.1)$ \\
$\cdot$ Saw Scaled Viper & 0 & $3(0.3)$ \\
$\cdot$ Green pit Viper & $28(2.7)$ & $33(2.8)$ \\
$\cdot$ Non venomous bite & $539(52.8)$ & $669(57.4)$ \\
$\cdot$ Unidentified bites & 1021 & 1165 \\
Total & & \\
\hline
\end{tabular}


Table 3

Baseline characteristics of co-morbidities of patients with snakebites in the study hospitals in Kurunegala district.

\begin{tabular}{|lll|}
\hline Type of Medical Comorbidities* & $\begin{array}{l}\text { Intervention } \\
\mathbf{n}(\%)\end{array}$ & $\begin{array}{l}\text { Control } \\
\mathbf{n}(\%)\end{array}$ \\
\hline Hypertension & $47(4.6)$ & $46(4)$ \\
\hline Diabetes mellitus & $21(2)$ & $28(2.4)$ \\
\hline Ischemic Heart Diseases & $3(0.3)$ & $8(0.7)$ \\
\hline Choric Kidney Diseases & $4(0.4)$ & $2(0.2)$ \\
\hline Hyperlipidemia & $3(0.3)$ & $4(0.3)$ \\
\hline Osteo/Rheumatoid Arthritis & $1(0.1)$ & $2(0.2)$ \\
\hline Bronchial Asthma & $15(1.5)$ & $25(2)$ \\
\hline Other lung Infection's diseases & $2(0.2)$ & $1(0.1)$ \\
\hline Known allergy to either medications or food & $18(1.8)$ & $23(2)$ \\
\hline Anemia and blood disorders & $2(0.2)$ & $4(0.3)$ \\
\hline Hypothyroidisms & $3(0.3)$ & $1(0.1)$ \\
\hline GoRD & $1(0.1)$ & $1(0.1)$ \\
\hline Carcinoma (Oral, Breast) & - & $2(0.2)$ \\
\hline Epilepsy & $2(0.1)$ & $3(0.3)$ \\
\hline Past history of Snakebites & $1(0.1)$ & $3(0.3)$ \\
\hline Psychiatric illness & $2(0.2)$ & $4(0.3)$ \\
\hline Pregnancy/Lower segment caesarean section (LSCS) & $1(0.1)$ & $3(0.3)$ \\
\hline Past surgical histories related to other medical conditions & $2(0.2)$ & $2(0.2)$ \\
\hline Other medical conditions & & $4(0.3)$ \\
\hline$*$ The total number of Co- morbidities were reported either as sole manifestations or in combination \\
\hline
\end{tabular}


Table 4

Hospital outcomes of snakebite patients admitted to primary Hospitals in Kurunegala District in North Western Province of Sri Lanka

\begin{tabular}{|lll|}
\hline Primary hospital outcomes of the snakebite admissions & $\begin{array}{l}\text { Intervention } \\
\mathbf{n}(\%)\end{array}$ & $\begin{array}{l}\text { Control } \\
\mathbf{n}(\%)\end{array}$ \\
\hline Discharged & $680(67)$ & $742(64)$ \\
Transferred & $208(20)$ & $191(16)$ \\
Death & 0 & 0 \\
Left the hospital before discharge decision and went home. & $133(13)$ & $232(20)$ \\
Total & 1021 & 1165 \\
\hline
\end{tabular}

Table 5

Details of the numbers analyzed by cluster and Intraclass correlation coefficients.

\section{Table5:}

\begin{tabular}{llllll} 
Primary Outcome Variables & Intervention & Control & ICC & $\begin{array}{l}\text { Adj.test } \\
\text { stat }\end{array}$ & $\begin{array}{l}\text { p- } \\
\text { value }\end{array}$ \\
& group & group & & & \\
$\begin{array}{l}\text { 04) Quality of Medical } \\
\text { Records }\end{array}$ & 20 & 17 & $-0.5486^{\mathrm{b}}$ & 0.5833 \\
\hline
\end{tabular}

No. Hospitals

13 (IQR:11.5- 13 (IQR 11-

Snakebite patients admitted 14.5) 15)

- Median scores

05) Appropriateness of Transfers

No. Hospitals

- Transfer indicated

06) Overall patient management ${ }^{*}$

No. Hospitals

- Satisfactory **
18

$32 / 208$

0.0252

$0.2^{c}$

0.6823

26/191

20

17

0.1016

$0.4^{\mathrm{C}}$

0.5032

$852 / 933$

a- Intra-cluster correlation (Anova estimate); b-Clustered Wilcoxon rank sum test; c-ICC Adjusted chisquared test; ${ }^{*}$ The patients who left the hospital early before discharge decisions were excluded from this assessment, ${ }^{\star *}$ If the patient correctly managed as per SLMA guideline

\section{Figures}




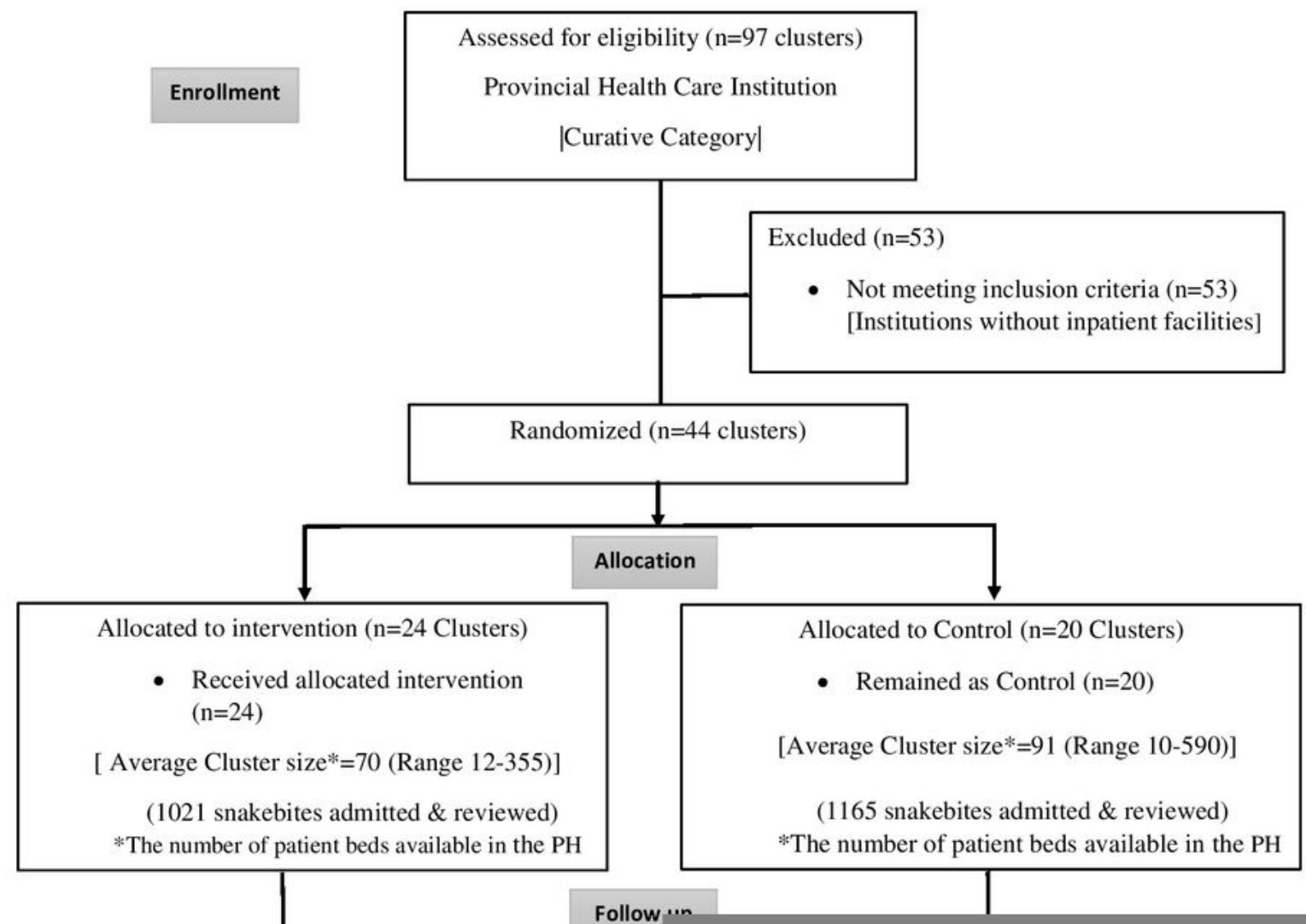

\section{Figure 1}

Consort flow diagram - Recruitment flow chart of clusters and participants; A hospital based educational intervention program to improve the snakebite management in Primary Hospitals. 
Quality of Medical Record-Score

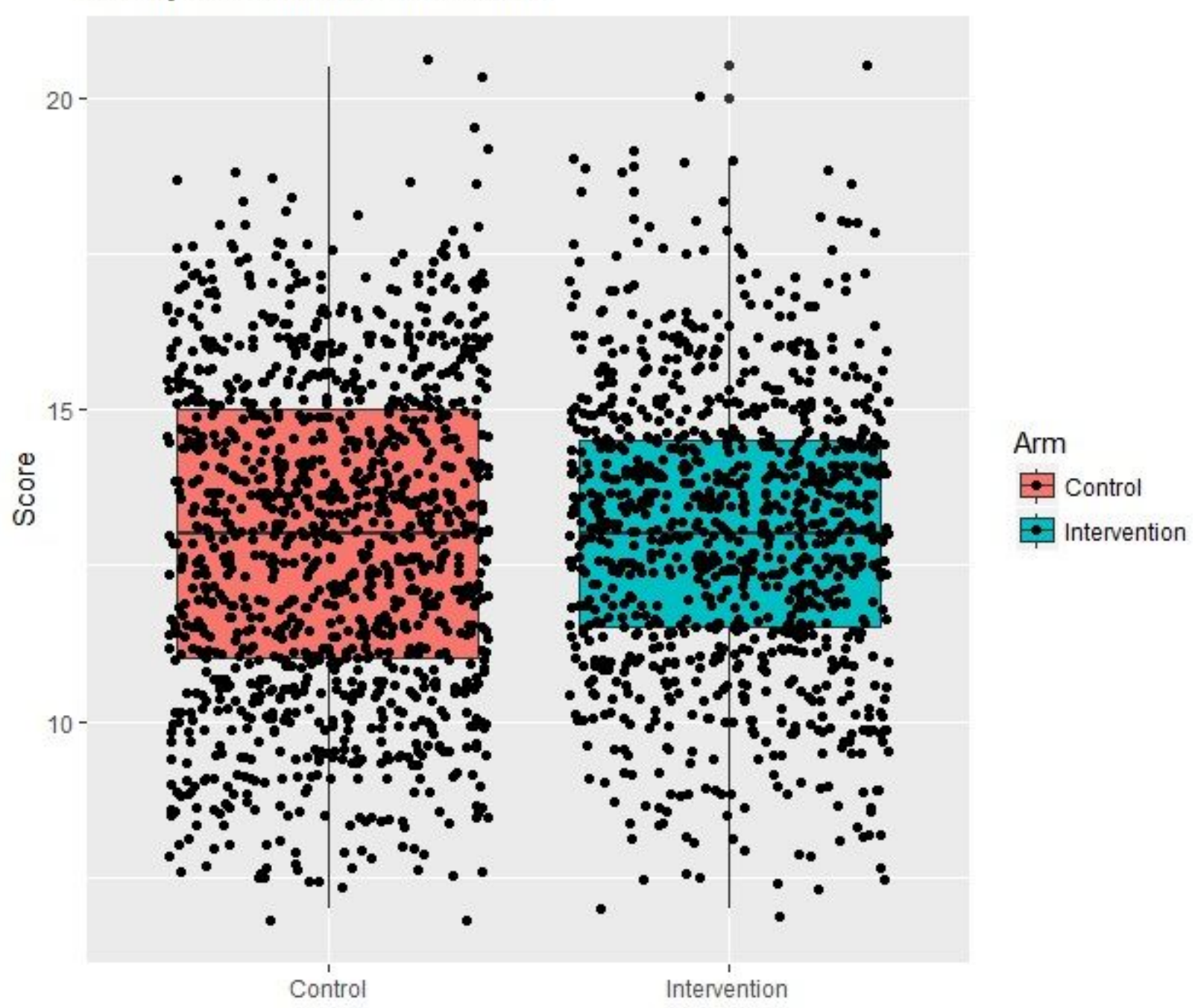

Figure 2

Comparison of two groups-scores for quality of recording in hospital records for $\mathbf{2 4}$ pre-determined points-scatter diagram. 
Total transfers $(n=399)$

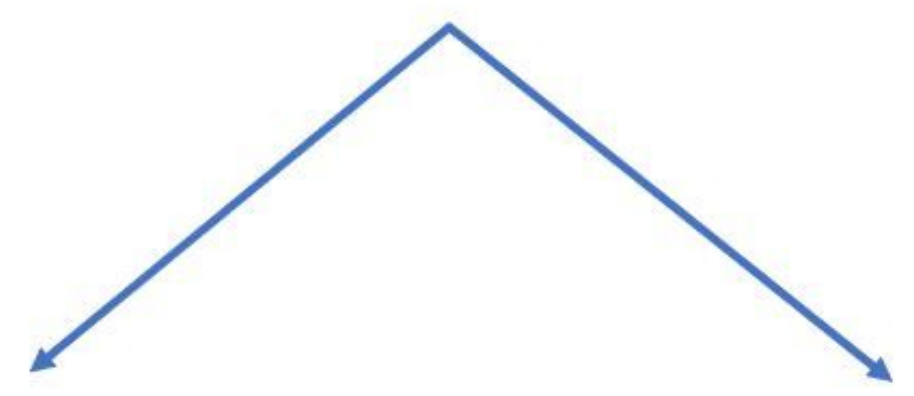

Intervention hospitals $(n=208)$
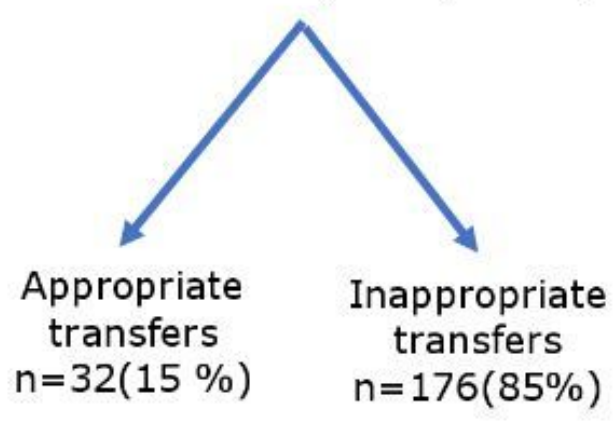

Control hospitals $(n=191)$

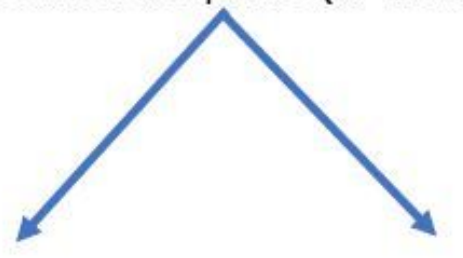

Appropriate

transfers

$n=26(14 \%)$

Inappropriate transfers

$n=165(86 \%)$

\section{Figure 3}

Flow chart diagram- Total number of transferred patients; appropriate vs inappropriate transfers 
For subsequent analysis: the number of case records of the transferred patients to THK available $(n=177)$

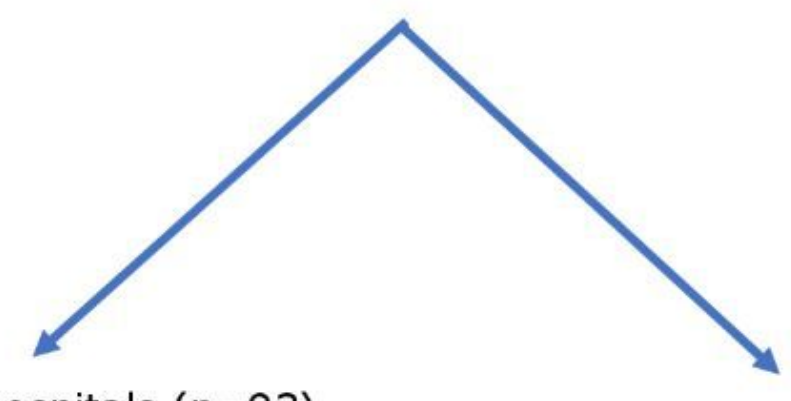

Intervention hospitals $(n=93)$

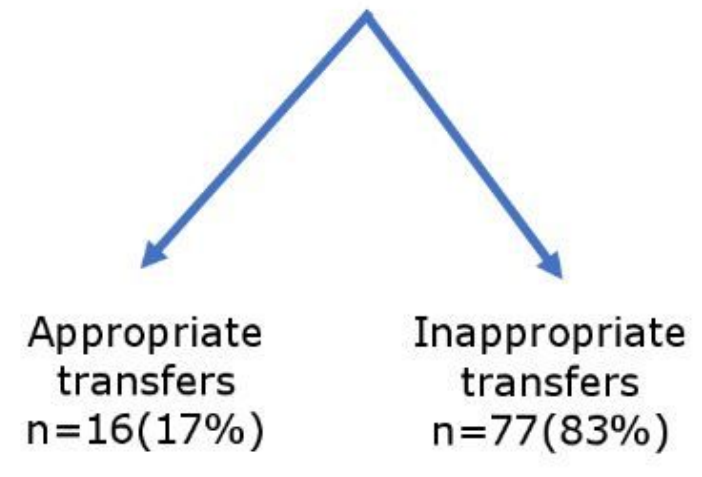

Control hospitals $(n=84)$
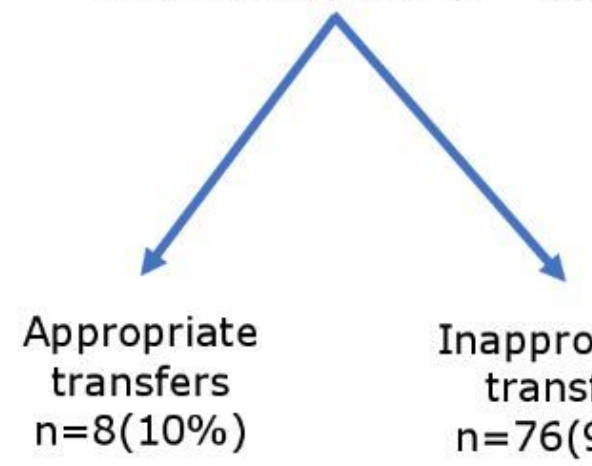

\begin{abstract}
Inappropriate
transfers

$n=76(90 \%)$
\end{abstract}

\section{Figure 4}

Flow chart diagram-Subsequent analysis of Transferred patients to tertiary care unit Teaching Hospital Kurunegala (THK); appropriate vs inappropriate

\section{Supplementary Files}

This is a list of supplementary files associated with this preprint. Click to download.

- Table1.jpg

- AdditionalFile1BHTfolio.pdf

- AdditionalFile2dataextractionForm.pdf

- AdditionalFile3PredeterminedPoints.pdf

- AdditionalFile4Protocol.pdf

- AdditionalFile5CONSORTChecklistClusterRCTeducationallntervention.docx

- AdditionalFile6datasetRCTscore.csv

- AdditionalFile7datasetTransfer.csv

- AdditionalFile8datasetManaged.csv

- AdditionalFile9RscriptDonnerTestClusrank.docx 